\title{
CIENCIAMATRIA
}

Revista Interdisciplinaria de Humanidades, Educación, Ciencia y Tecnología

Año VII. Vol. VII. N¹2. Enero - Junio. 2021

Hecho el depósito de ley: pp201602FA4721

ISSN-L: 2542-3029; ISSN: 2610-802X

Universidad Nacional Experimental Francisco de Miranda (UNEFM). Santa Ana de Coro. Venezuela

Janeth Gabriela Molina-Romo; Katina Vanessa Bermeo-Pazmiño

Diego Patricio Cisneros-Quintanilla

$\underline{\text { DOI } 10.35381 / \mathrm{cm} . v 7 i 12.425}$

\section{Modelo de gestión estratégica de Talento Humano para el Instituto Ecuatoriano de Seguridad Social}

\section{Strategic Management Model of Human Talent for the Ecuadorian Institute of Social Security}

\author{
Janeth Gabriela Molina-Romo \\ janeth.molina.58@est.ucacue.edu.ec \\ Universidad Católica de Cuenca, Cuenca \\ Ecuador \\ https://orcid.org/0000-0002-4072-6119 \\ Katina Vanessa Bermeo-Pazmiño \\ kbermeo@ucacue.edu.ec \\ Universidad Católica de Cuenca, Cuenca \\ Ecuador \\ https://orcid.org/0000-0002-4438-7855 \\ Diego Patricio Cisneros-Quintanilla \\ dcisneros@ucacue.edu.ec \\ Universidad Católica de Cuenca, Cuenca \\ Ecuador \\ https://orcid.org/0000-0003-0897-8938
}

Recibido: 01 de octubre de 2020

Aprobado: 15 de diciembre de 2020 


\author{
CIENCIAMATRIA \\ Revista Interdisciplinaria de Humanidades, Educación, Ciencia y Tecnología \\ Año VII. Vol. VII. N¹2. Enero - Junio. 2021 \\ Hecho el depósito de ley: pp201602FA4721 \\ ISSN-L: 2542-3029; ISSN: 2610-802X \\ Universidad Nacional Experimental Francisco de Miranda (UNEFM). Santa Ana de Coro. Venezuela \\ Janeth Gabriela Molina-Romo; Katina Vanessa Bermeo-Pazmiño \\ Diego Patricio Cisneros-Quintanilla
}

\begin{abstract}
RESUMEN
La investigación tuvo por objetivo diseñar un modelo de gestión estratégica de Talento Humano para el Instituto Ecuatoriano de Seguridad Social Azogues - Ecuador. Se generó desde una tipología metodológica descriptiva con diseño no experimental. Se evidenció que no existe compensación a los funcionarios, las capacitaciones no se realizan en función a las estratégicas y necesidades institucionales, influyen de manera directa con el cumplimiento de los procesos organizaciones que encadena retraso en el desarrollo de competencias individuales. Este modelo de gestión estratégica aportará en la productividad eliminando las fallas en los procesos y optimizando los recursos. En el proceso de aplicación de las encuestas se constató que el personal reconoce a la Unidad Administrativa de Talento Humano como un proceso estratégico, sin embargo, no cubre por completo sus necesidades. Se evidenció que la Institución, posee una estructura organizacional definida, bajo lo cual desarrolla los procesos.
\end{abstract}

Descriptores: Administración financiera; gestión de recursos; gestión del personal. (Palabras tomadas del Tesauro UNESCO).

\begin{abstract}
The objective of the research was to design a strategic management model of Human Talent for the Ecuadorian Institute of Social Security Azogues - Ecuador. It was generated from a descriptive methodological typology with a non-experimental design. It was evidenced that there is no compensation for officials, training is not carried out according to the strategic and institutional needs, they directly influence the fulfillment of the organizational processes that lead to a delay in the development of individual competencies. This strategic management model will contribute to productivity by eliminating failures in processes and optimizing resources. In the process of applying the surveys, it was found that the staff recognizes the Human Talent Administrative Unit as a strategic process, however, it does not fully cover their needs. It was evidenced that the Institution has a defined organizational structure, under which it develops the processes.
\end{abstract}

Descriptors: Financial administration; resources management; personnel management. (Words taken from the UNESCO Thesaurus). 


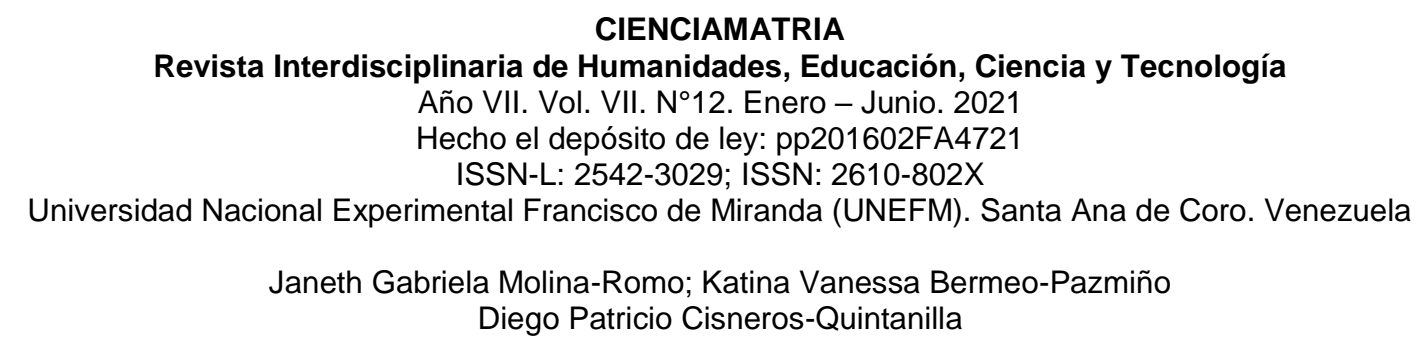

\section{INTRODUCCIÓN}

Con el avance de procesos actuales organizativos y la búsqueda de varios aspectos que respondan a una buena gestión a través de criterios de calidad, compromiso, ética, organización, participación individual como colectiva, han logrado establecer planes, procesos y modelos de gestión que mejoren el desarrollo organizacional.

En la actualidad podemos encontrar varios modelos de gestión, entre ellos están; gestión estratégica, gestión por resultados, gestión por procesos, gestión del talento humano, gestión del conocimiento y gestión por competencias. Este descubrimiento, desarrollo y puesta en práctica de los modelos hacen que se conviertan en fuentes esenciales de guía con el que se puede obtener una visión sistémica en las organizaciones.

En el sector público se ha reconocido el papel estratégico que aporta el talento humano en el fortalecimiento de valores institucionales, a causa de los cambios del entorno y cultura de la sociedad. La respuesta que refleje cada institución a estos cambios hará que se adapte más fácilmente, que se generen ideas y soluciones acertadas, proporcione desarrollo de procesos de manera ágil, se elimine cuellos de botella y así responda a varias situaciones del entorno.

El conocimiento de los colaboradores de la institución son la pieza clave para contestar a los constantes cambios, con resultados positivos, siendo fundamental mantener a los colaboradores capacitados con la finalidad de formar la base de la innovación y creación de estructuras sólidas, trascendental y estratégico para el desarrollo de la organización. Es importante que la gestión del talento humano deba sostener una visión al futuro, para prever cambios y evaluar las consecuencias de los mismos, para retransmitir esta visión a todos los colaboradores a través del liderazgo, actuando en conjunto, sembrando bases sólidas en trabajo en equipo para promover la co-innovación, desarrollando el aprendizaje por medio de conocimientos multidisciplinarios (Barrios-Hernández, et al., 2020).

Es importante señalar que la gestión del talento humano también permite que las instituciones mejoren el desempeño de los colaboradores, siendo primordial el desarrollo 


\section{CIENCIAMATRIA \\ Revista Interdisciplinaria de Humanidades, Educación, Ciencia y Tecnología \\ Año VII. Vol. VII. N¹2. Enero - Junio. 2021 \\ Hecho el depósito de ley: pp201602FA4721 \\ ISSN-L: 2542-3029; ISSN: 2610-802X \\ Universidad Nacional Experimental Francisco de Miranda (UNEFM). Santa Ana de Coro. Venezuela \\ Janeth Gabriela Molina-Romo; Katina Vanessa Bermeo-Pazmiño \\ Diego Patricio Cisneros-Quintanilla}

de las estrategias y herramientas, para así con un modelo de gestión del talento humano contribuir al mejoramiento de procesos internos, disminuir tiempo, optimizar recursos y así llegar a la "meta" que es la planificación estratégica definida por la organización. El modelo de (Harper y Lynch, 1992) como citó (Pico, 2016), señala que partiendo del plan estratégico se elabora la proyección de necesidades de talento humano que incluye: planificación de talento humano, selección de personal, formación y capacitación, evaluación de desempeño, motivación, entre otras. Con estos subsistemas se logrará una optimización del talento humano, siempre y cuando exista seguimiento a los procesos que van de la mano con los resultados alcanzados para la consecución de objetivos.

En este contexto el Instituto Ecuatoriano de Seguridad Social es una entidad, cuya organización y funcionamiento se fundamenta en los principios de solidaridad, obligatoriedad, universalidad, equidad, eficiencia, subsidiariedad y suficiencia. Se encarga de aplicar el Sistema del Seguro General Obligatorio que forma parte del sistema nacional de Seguridad Social (Ley Orgánica de Seguridad Social, 2011).

Cabe destacar que esta Institución cuenta con 101 unidades médicas que se clasifican por su nivel de complejidad, enlazadas todas al plan estratégico Institucional que permite a las autoridades y servidores encaminar todos sus esfuerzos para alcanzar la misión y visión del IESS. En muchas ocasiones se ha observado la falta de liderazgo en la Dirección, que repercute en la gestión estratégica del talento humano, ocasionando que los procesos no sean claros, impidiendo que el recurso humano este armonizado con las funciones desempeñadas y estas a su vez con los objetivos estratégicos Institucionales. Esta propuesta está dirigida a mejorar el modelo de gestión estratégica de talento humano ajustado a la problemática actual y que fortalezca los procesos existentes, a través de una guía conformada por varias técnicas que mejoren el desempeño laboral de los empleados lo que permitirá alcanzar los objetivos planteados en el plan estratégico del IESS. 


\section{CIENCIAMATRIA \\ Revista Interdisciplinaria de Humanidades, Educación, Ciencia y Tecnología \\ Año VII. Vol. VII. N¹2. Enero - Junio. 2021 \\ Hecho el depósito de ley: pp201602FA4721 \\ ISSN-L: 2542-3029; ISSN: 2610-802X \\ Universidad Nacional Experimental Francisco de Miranda (UNEFM). Santa Ana de Coro. Venezuela \\ Janeth Gabriela Molina-Romo; Katina Vanessa Bermeo-Pazmiño \\ Diego Patricio Cisneros-Quintanilla}

A través del esquema de modelo de gestión del talento humano se fortalecerá el desempeño de los servidores públicos del CRIE Azogues, es necesario tomar la importancia que amerita en la planificación, selección de personal y su contratación, capacitación y formación profesional y evaluación de desempeño.

En tal sentido, la investigación tuvo por objetivo diseñar un modelo de gestión estratégica de Talento Humano para el Instituto Ecuatoriano de Seguridad Social Azogues Ecuador.

\section{Referencial Teórico}

\section{La Gestión del Talento Humano en el sector público salud}

La gestión del talento humano en la organización y, en general, los recursos humanos han sufrido cambios drásticos, tiempo atrás los departamentos de recursos humanos estaban encaminados a funciones estáticas, como contrataciones, nóminas, beneficios, control de personal; en la actualidad, las organizaciones comienzan a entender y valorar el impacto positivo de mantener un equipo de colaboradores calificados, comprometidos e involucrados con la planificación estratégica de la organización (Armijos-Mayon, et al., 2019).

El conjunto de procesos de la organización, diseñados para captar, gestionar, desarrollar, motivar y mantener a los colaboradores se denomina gestión de talento humano. La obtención de mejores resultados de la institución con la colaboración de cada uno de los empleados es la base para que se logre la planificación estratégica, fomentando el desarrollo profesional de los colaboradores, el enfoque humano y el logro de metas organizacionales. Se debe indicar que la gestión del talento humano no se realiza exclusivamente desde la unidad de talento humano, pero sí se encuentra en ella sus cimientos; el resultado esperado está relacionada con la posibilidad de aportar o fortalecer las capacidades de la Institución (Ulrich, 2000). 


\section{CIENCIAMATRIA \\ Revista Interdisciplinaria de Humanidades, Educación, Ciencia y Tecnología \\ Año VII. Vol. VII. N¹2. Enero - Junio. 2021 \\ Hecho el depósito de ley: pp201602FA4721 \\ ISSN-L: 2542-3029; ISSN: 2610-802X \\ Universidad Nacional Experimental Francisco de Miranda (UNEFM). Santa Ana de Coro. Venezuela \\ Janeth Gabriela Molina-Romo; Katina Vanessa Bermeo-Pazmiño \\ Diego Patricio Cisneros-Quintanilla}

La Unidad de Talento Humano debe ser un libro abierto de información, al que puedan acceder todas las áreas de la Institución, con la finalidad de conocer los objetivos y planes estratégicos. Las organizaciones que se proponen desarrollar y fortalecer su talento humano, adaptan planes y proyectos, sin descuidar el seguimiento para evaluar resultados. Para administrar el talento humano de mejor manera se realiza lo siguiente:

1. Hallar, convencer y reclutar a los aspirantes que cumplen los requisitos de manera competitiva.

2. Administrar y establecer sueldos de acuerdo con cada cargo.

3. Aprendizaje continuo a través de la formación y capacitación.

4. Definir procedimientos para la evaluación del desempeño.

5. Motivar y fomentar el buen clima laboral.

6. Mantener programas de ascensos y traslados (Chiavenato, 2004)

El desarrollo competitivo de mediano y largo plazo, para definir objetivos, elaborar estrategias y determinar acciones que resulten en aumento de la competitividad, asegurando la sostenibilidad de la organización se logra con el proceso de planeación estratégica. El desarrollo de la planeación estratégica es la herramienta que encuentra y especifica ventajas competitivas de la organización, para alcanzar los objetivos organizacionales (Chiavenato \& Sapiro, 2017).

La gestión de talento humano "es un enfoque estratégico de dirección cuyo objetivo es obtener la máxima creación de valor para la organización, a través de un conjunto de acciones dirigidas a disponer en todo momento del nivel de conocimientos, capacidades y habilidades en la obtención de los resultados necesarios para ser competitivo en el entorno actual y futuro" (Quesada-Quecan, 2014).

Busca el involucramiento de los colaboradores, maximizando las competencias de cada individuo que forma parte de una institución, la GTH proporciona comunicación entre empleadores y empleados con el fin de ayudarlos, respaldarlos y ofrecerles un desarrollo 


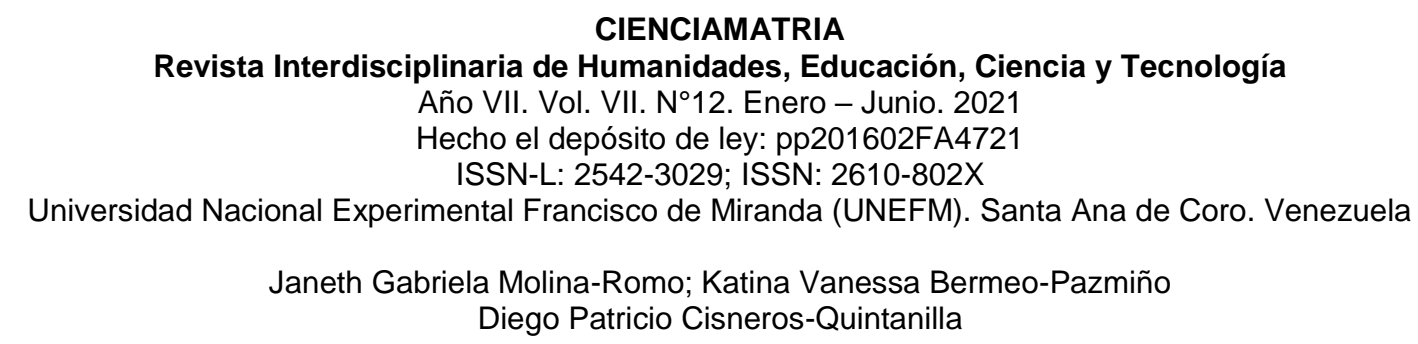

personal capaz de enriquecer la personalidad y motivación de cada empleado que representa lo más importante de la organización (Chávez, 2015).

El objetivo está en llegar al talento humano de tal manera que sea capaz de convertirse en una ventaja empresarial (competitividad y productividad), de vital importancia para la organización, pues si "los colaboradores están dispuestos a entregar todo su esfuerzo, la organización marchará; caso contrario, se estancará", es decir, el talento humano directamente influye de manera proactiva a los objetivos organizacionales (Chávez, 2015).

La GTH depende, entre otros, de varios aspectos como son: cultura organizacional, la estructura organizacional de la Institución, la sociedad, la misión de la institución, la tecnología y los procesos internos (Chiavenato, 2002). Analizando cada uno de los aspectos la gestión de talento humano está conformada por colaboradores y la Institución, la administración del talento humano debe perseguir la optimización de habilidades de cada uno, la integración, la creatividad, la formación. Los funcionarios y trabajadores deben ser considerados como el activo más relevante en la organización, así entonces, la gestión de talento humano conlleva al reconocimiento de los colaboradores como capaces de proporcionar a la organización de inteligencia y llevarla a la excelencia (Chiavenato, 2002). Siendo básico conocer un breve resumen de cada uno de los procesos de la moderna gestión del talento humano que son (Cerón- Gordón, et al., 2017):

1. Admisión de personas: mecanismo que se usa para incluir nuevos funcionarios en la institución que nacen a través de una necesidad, incluyen reclutamiento, selección y contratación de personal.

2. Aplicación de personas: procedimiento usado para crear actividades que los colaboradores realizarán en la organización, que servirá de base para su evaluación de desempeño. En este proceso se puede incorporar el diseño organizacional, análisis y descripción de cargos, y evaluación del desempeño. 
3. Compensación del personal: es el incentivo remunerativo, de recompensas o beneficios que perciben los colaboradores.

4. Desarrollo del personal: permite a los colaboradores la generación de conocimientos científicos con la finalidad de incrementar el desarrollo profesional y personal. Se puede incluir en este proceso el entrenamiento y desarrollo de las personas, programas de desarrollo de las carreras y de integración entre otras.

5. Servicios de Personal: este proceso tiene que ver con las condiciones en las que se desenvuelve el colaborador en la institución, también se podría incluir en este proceso a la seguridad e higiene en el trabajo, y mantenimiento de relaciones sindicales.

6. Evaluación del personal: procedimiento por el cual se planificará y desarrollará la evaluación de desempeño de los colaboradores con el fin de estimular su rendimiento y obtener resultados de las actividades que cada uno desempeña.

En el sector público la unidad administrativa del talento humano será quien ejerza atribuciones y responsabilidades otorgadas por la Ley Orgánica de Servicio Público LOSEP relacionados con la administración del personal. El subsistema de planificación de talento humano según señala la Ley Orgánica de Servicio Público" Es el conjunto de normas, técnicas y procedimientos orientados a determinar la situación histórica, actual y futura del talento humano, a fin de garantizar la cantidad y calidad de este recurso, en función de la estructura administrativa correspondiente" (Ley Orgánica del Servicio Público, 2016).

En el sector público existen dos tipos de regímenes LOSEP y Código de Trabajo, para los funcionarios bajo la Ley Orgánica de Servicio Público el sistema integrado de desarrollo del talento humano se basa en: subsistema de planificación del talento humano subsistema de clasificación de puestos, subsistema de selección de personal, subsistema de formación y capacitación y el subsistema de evaluación del desempeño (Ley Orgánica del Servicio Público, 2016). 


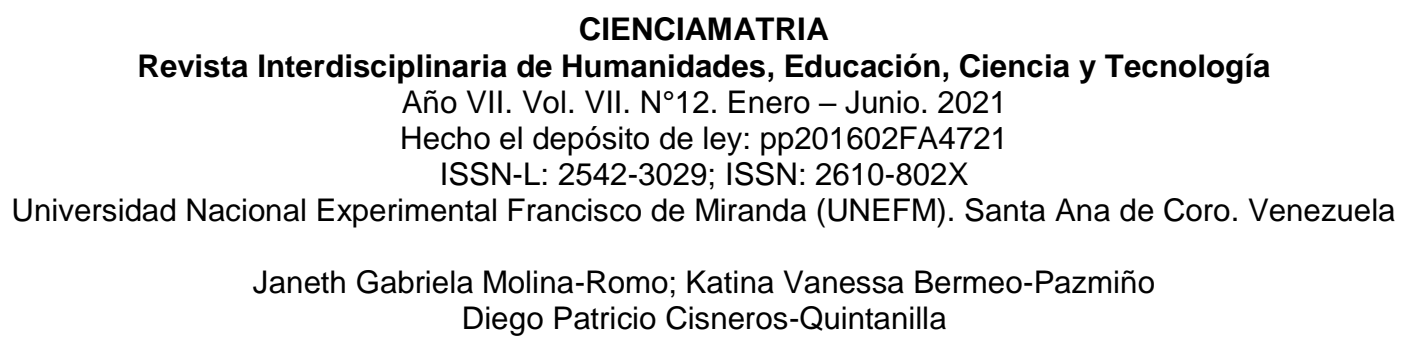

\section{Planeación Estratégica en la administración de talento humano}

Al hablar de planeación estratégica de talento humano hacemos referencia a una organización proactiva no reactiva en los resultados que desea encaminarse a futuro. A largo plazo debe ser un proceso flexible, integral, completo que genera capacidad de dirección. Es importante señalar que se debe involucrar a esta planeación estratégica el entorno externo e interno, para lograr adaptarse a los cambios generando valor a la organización (Zuin-Secco, et al., 2016).

Se ha podido evidenciar que al pasar del tiempo la gestión se vuelve más competitiva y que los desafíos aumentan, es indispensable poseer una planeación estratégica, ya que es un proceso básico en toda institución sea pública o privada que se encarga de diseñar los objetivos a ser alcanzados por cada una de las unidades que forman parte de una organización y a su vez a través de la Dirección emitir las directrices a seguir para definir los planes de acción, sin descuidar la generación de ventajas competitivas, sostenibilidad a largo plazo, que serán reflejados en los resultados alcanzados.

La planificación de recursos humanos abarca el estudio cuantitativo y cualitativo de las necesidades de recursos humanos que la organización puede tener en el corto, mediano como en el largo plazo. La planeación permite contrastar las capacidades internas actuales con las necesidades presentes y futuras de la organización identificando las acciones que deben emprenderse para cubrir dichas necesidades (Delaux, 2017), así mismo, Chiavenato (2004) afirma que "la administración es el proceso de planear, organizar, dirigir y controlar el uso de los recursos para lograr los objetivos organizacionales" (p. 10).

La persona que se encuentra en el proceso de talento humano deberá tener la capacidad de entender, comprender, actuar de manera inteligente para resolver diversos casos que se suscitan en el diario vivir, demostrando: habilidades, destrezas, experiencias, personalidad, aptitudes propias de cada ser. Paralelamente esta capacidad es favorable cuando otros factores o talentos se encuentran presentes en el ser humano como: 
conocimiento, potencial, competencias adquiridas, experiencias, motivación, vocación, ánimo, salud, (Cerón-Gordón, et al., 2017).

En una organización es importante lograr la motivación, para explotar al máximo sus habilidades, destrezas, conocimiento, de esta manera se obtendrá el alcance de la planeación estratégica en beneficio de la Institución. Por otro lado, Alles (2008) indica que:

La motivación de las personas en el ámbito laboral es un tema estudiado en profundidad por diferentes autores, desde hace mucho tiempo, sin que por ello pierda actualidad. Las organizaciones se encuentran frecuentemente preocupadas y los diseños de soluciones no siempre dan el resultado esperado (p. 33)

Existe el concepto errado de la motivación hacia los colaboradores, cuando los directivos actúan y realizan actividades que ellos necesitan "creen" que están motivados, sin embargo, se motivan cuando en realidad hacen lo que desean. Sin motivación una organización no funciona. La motivación en el trabajo es como el combustible para un motor. La motivación es esencial para el buen funcionamiento de una organización. Una organización puede tener la mejor tecnología y equipamiento, pero si su personal no se siente motivado tales recursos serán improductivos (Marín-Samanez \& PlacenciaMedina, 2017).

La organización a través del departamento de talento humano requiere captar aquellos comportamientos y talentos relevantes en los colaboradores para aprovecharlos en el cumplimiento de objetivos por ende la planificación de la organización. Los comportamientos pueden ser medidos en términos de competencias de cada individuo y su nivel de aporte a la organización. La Administración del desempeño según PérezMontejo (2009) es definida como:

el proceso mediante el cual la compañía asegura que el empleado trabaja alineado con las metas de la organización, así como las prácticas a través de las cuales el trabajo es definido y revisado, las capacidades son desarrolladas y las recompensas son distribuidas en las organizaciones 


\section{CIENCIAMATRIA \\ Revista Interdisciplinaria de Humanidades, Educación, Ciencia y Tecnología \\ Año VII. Vol. VII. No12. Enero - Junio. 2021 \\ Hecho el depósito de ley: pp201602FA4721 \\ ISSN-L: 2542-3029; ISSN: 2610-802X \\ Universidad Nacional Experimental Francisco de Miranda (UNEFM). Santa Ana de Coro. Venezuela \\ Janeth Gabriela Molina-Romo; Katina Vanessa Bermeo-Pazmiño \\ Diego Patricio Cisneros-Quintanilla}

La evaluación de desempeño permite adoptar o retroalimentar políticas de compensación y beneficios para premiar a los colaboradores en su desempeño (ascensos, reubicación, preseas, etc.), o al contario determinar los cuellos de botella para actuar directamente en el estancamiento de procesos que impiden que la planeación se realice correctamente. Se tomaría la decisión si fuera el caso de volver a capacitar, detectar errores en el diseño del puesto, asignación de funciones, o si los resultados de la evaluación de desempeño arrojan problemas personales que afecten al colaborador en el desempeño de funciones (Stoner \& Wankel, 1990).

\section{Capacitación y Desarrollo del Personal}

Para Chiavenato (2011), la capacitación "es la obtención de conocimientos de corto plazo, aplicado de manera sistemática y organizada, a través de este las personas adquieren conocimientos, desarrollan habilidades y competencias en función de objetivos definidos", desde ese punto de vista es importante definir cuatro pasos para realizar una capacitación:

1. Levantamiento de necesidades de capacitación a través de un diagnostico por áreas.

2. Definir el plan anual de capacitación para satisfacer las necesidades de cada área.

3. Desarrollo y ejecución del plan anual de capacitación.

4. Análisis y retroalimentación de resultados de la evaluación. (Cerón-Gordón., 2017) Se destaca la finalidad de la capacitación de personal:

1. Invertir en los colaboradores con el objetivo de prepararlos en diversas tareas y retos del cargo.

2. Colocar diversas oportunidades de desarrollo personal, ya sea para el puesto actual o futuro considerando en el segundo funciones más complejas.

3. Motivar a los colaboradores a cambiar su actitud para crear o mejorar un clima laboral que aumente las expectativas laborales. 


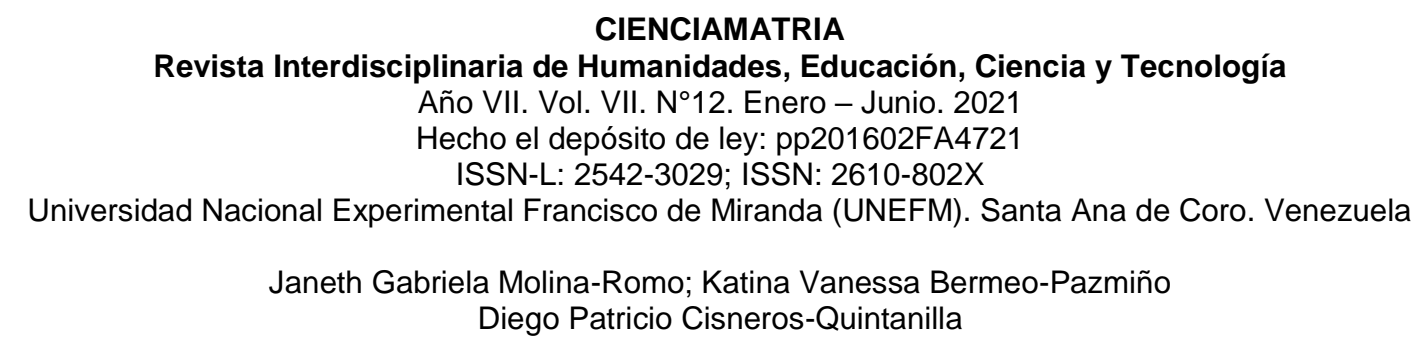

\section{Modelos de Gestión Estratégica de Talento Humano}

El Modelo de gestión estratégica de Recursos Humanos desarrolla distintas partes integrantes siendo la "Estrategia" el gran desarrollo que fija el rumbo de la organización y en base a esta se define la "Planificación", podemos encontrar como principales lineamientos a la organización de trabajo, la gestión de empleo, rendimiento, compensación, presupuesto de gasto de recursos humanos y gestión de la administración.

Las instituciones públicas acostumbran a gestionar las retribuciones de forma inadecuada. En general los modelos que se implementan son rígidos, uniformes basados en categorías, puestos y antigüedades. Además, existen variables que condicionan la gestión, como variable externa se puede considerar a las políticas gubernamentales y sindicales, tecnología, otros, y las variables internas que puede ser la estructura el tamaño la situación financiera (Bustos-Farías, et al., 2016). El modelo de gestión deberá estar alienado a los objetivos estratégicos de la organización. Generar compromiso en la organización a través de ventajas competitivas en mejora de costos y productividad construyendo valor y motivación en los colaboradores.

Los modelos de gestión de recursos humanos como la gestión de las organizaciones, sin duda ha cambiado en la actualidad debido a los grandes retos que se enfrenta, ocasionando la necesidad de sistemas de gestión cada vez más técnicos y avanzados.

En las filosofías gerenciales, indica (Aduna-Mondragón, et al., 2017), el desarrollo de las tecnologías de la información y las comunicaciones han aportado a la realización de un desempeño de actividades de manera ágil, inteligente para el logro de las ventajas competitivas sustentables de la organización, para ahorrar tiempo y recursos es necesario optimizar el talento humano a través de un modelo de GRH.

En la búsqueda de modelos técnicos, funcionales, aplicables para alcanzar a nueva concepción integral del funcionamiento del capital humano en las organizaciones se encuentran los siguientes: 


\section{Modelo de Besseyre DesHorts}

Hace referencia a un modelo de gestión estratégica de talento humano visualizado mediante los procesos generales que se realiza en el área de talento humano, asegurando la gestión de competencias a través de potenciar prácticas recién adquiridas, estimularlas y desarrollarlas formando la misión de la organización (Aduna- Mondragón, et al., 2017).

1. Adquirirlas menciona definición de puestos o funciones, con la finalidad de formar una base de perfiles de puestos.

2. Estimularlas a través de un sistema de retribuciones que mejoren las condiciones de trabajo de manera equitativa y motivadora.

3. Desarrollarlas que quiere decir formación personal para realizar un diagnóstico de la organización, con le fin de definir objetivos para lograr estrategias para el desarrollo del RH. (Aduna- Mondragón, et al., 2017).

4.

\section{Modelo de Beer y Colaboradores}

Para este modelo los autores consideran importante la influencia de los empleados en su actuar con el equipo de trabajo, además de las políticas de talento humano como: Sistema de trabajo, Flujo de RH y Sistemas de Recompensas (Aduna- Mondragón, et al., 2017).

\section{Modelo de Gestión de Talento Humano de Werther y Davis}

Este modelo indica que en la actualidad se necesita una administración de Talento Humano alineada con los objetivos estratégicos de la organización, debido a los grandes retos producidos por la globalización y tecnología (Werther, 2016).

Los Autores plantean lo siguiente:

Preparación y selección: Mantener y custodiar la información para certificar la eficiencia del talento humano. 


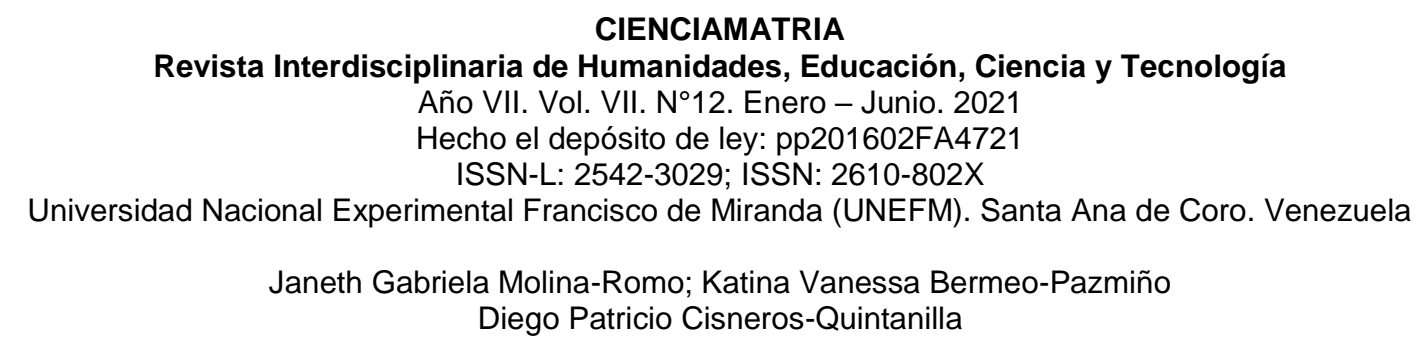

Desarrollo y evaluación: Con el objetivo de no perder los conocimientos brindados al personal a través de capacitaciones impartidas anteriormente, se debe elaborar un plan de capacitaciones constante para recordar la estrategia corporativa.

Compensación y protección: Básicamente hace referencia a la protección de los colaboradores en la Institución de riesgos físicos, psicológicos y otros.

Relación con el personal y evaluación: Evaluar los resultados obtenidos acorde a las metas proyectadas (Aduna- Mondragón, et al., 2017).

\section{MÉTODO}

La investigación se generó desde una tipología metodológica descriptiva con diseño no experimental, se realizó un muestreo por conveniencia que es un procedimiento no probabilístico al azar formado por el caso accesible y la disponibilidad de personas naturales que sean parte de la investigación, que, para el trabajo de investigación, se utilizó la encuesta y cuestionario como mecanismo de encontrar información al personal interno de la Institución 45 funcionarios del Instituto Ecuatoriano de Seguridad Social (IESS) Azogues-Ecuador.

\section{RESULTADOS}

Los principales resultados de la encuesta, dirigida a los funcionarios del Instituto Ecuatoriano de Seguridad Social son los siguientes:

Dimensión: Reclutamiento y Selección

Para esta dimensión, el $95.30 \%$ de los funcionarios y trabajadores encuestados señalan que el proceso de reclutamiento y selección realizada en la Unidad Médica cumple con las expectativas de cada cargo ya que se encuentran acorde al perfil profesional solicitado, el $4.70 \%$ indican que no existe una correcta valoración en los requisitos de experiencia y educación. 


\section{CIENCIAMATRIA \\ Revista Interdisciplinaria de Humanidades, Educación, Ciencia y Tecnología \\ Año VII. Vol. VII. No12. Enero - Junio. 2021 \\ Hecho el depósito de ley: pp201602FA4721 \\ ISSN-L: 2542-3029; ISSN: 2610-802X \\ Universidad Nacional Experimental Francisco de Miranda (UNEFM). Santa Ana de Coro. Venezuela \\ Janeth Gabriela Molina-Romo; Katina Vanessa Bermeo-Pazmiño \\ Diego Patricio Cisneros-Quintanilla}

Dimensión: Capacitación y formación

En esta dimensión, el $35.6 \%$ de los colaboradores encuestados consideran que ellos cumplen con las capacitaciones una vez al año, del plan de capacitaciones anuales del IESS, el $28,9 \%$ cada tres meses, el $22.2 \%$ cada seis meses, el $6.7 \%$ una vez al mes y el $6.7 \%$ no ha recibido.

Además, consideran el $57.8 \%$ de los encuestados que; se debe desarrollar la competencia de trabajo en equipo ya que señalan que es lo más importante para ser eficaz en su desempeño de trabajo, el 35.60\% indican que se requiere una capacitación constante de competencias técnicas del puesto y el $6.7 \%$ responden a que se requiere formación de todas las competencias.

Dimensión: Motivación

El $42,2 \%$ reconocen que la mayor motivación y satisfacción en su puesto de trabajo es la estabilidad laboral, el $22,2 \%$ de los funcionarios y trabajadores señalan que es fundamental el reconocimiento del trabajo, el $17.8 \%$ de los encuestados indica que influye el buen clima laboral y el $17.8 \%$ señala que se debe a las capacitaciones brindadas.

También se puede apreciar que el $44.4 \%$ de los encuestados se encuentran comprometidos con la Institución en el desarrollo de sus actividades, conocimientos y habilidades dentro de los plazos establecidos, el $40 \%$ de los funcionarios se encuentran motivados por la orientación al servicio y el $15.60 \%$ indican que se encuentran entusiastas para desarrollar las funciones.

Dimensión: Administración de Talento Humano

Según resultados de la encuesta aplicada se determina que el 59,10\% del personal opina que la gestión de Talento Humano es estratégica, mientras que el $40,90 \%$ opina es operativa, evidenciándose que la percepción de la gran mayoría de los trabajadores y 


\section{CIENCIAMATRIA}

Revista Interdisciplinaria de Humanidades, Educación, Ciencia y Tecnología

Año VII. Vol. VII. N¹2. Enero - Junio. 2021

Hecho el depósito de ley: pp201602FA4721

ISSN-L: 2542-3029; ISSN: 2610-802X

Universidad Nacional Experimental Francisco de Miranda (UNEFM). Santa Ana de Coro. Venezuela

Janeth Gabriela Molina-Romo; Katina Vanessa Bermeo-Pazmiño

Diego Patricio Cisneros-Quintanilla

empleados es que el área de Talento Humano se encuentra alineada a las estrategias del Instituto Ecuatoriano de Seguridad Social y de la dirección.

\section{PROPUESTA}

Esquema de propuesta "Modelo de gestión estratégica de Talento Humano en el Instituto Ecuatoriano de Seguridad Social"

Con este trabajo de investigación se pretende desarrollar un modelo de gestión estratégica de Talento Humano en el Instituto Ecuatoriano de Seguridad Social, planteado a través de un esquema de cuatro etapas secuenciales, que deben llevarse de manera armónica, organizada para cumplir con los parámetros y estrategias establecidas por la planificación estratégica de la Institución.

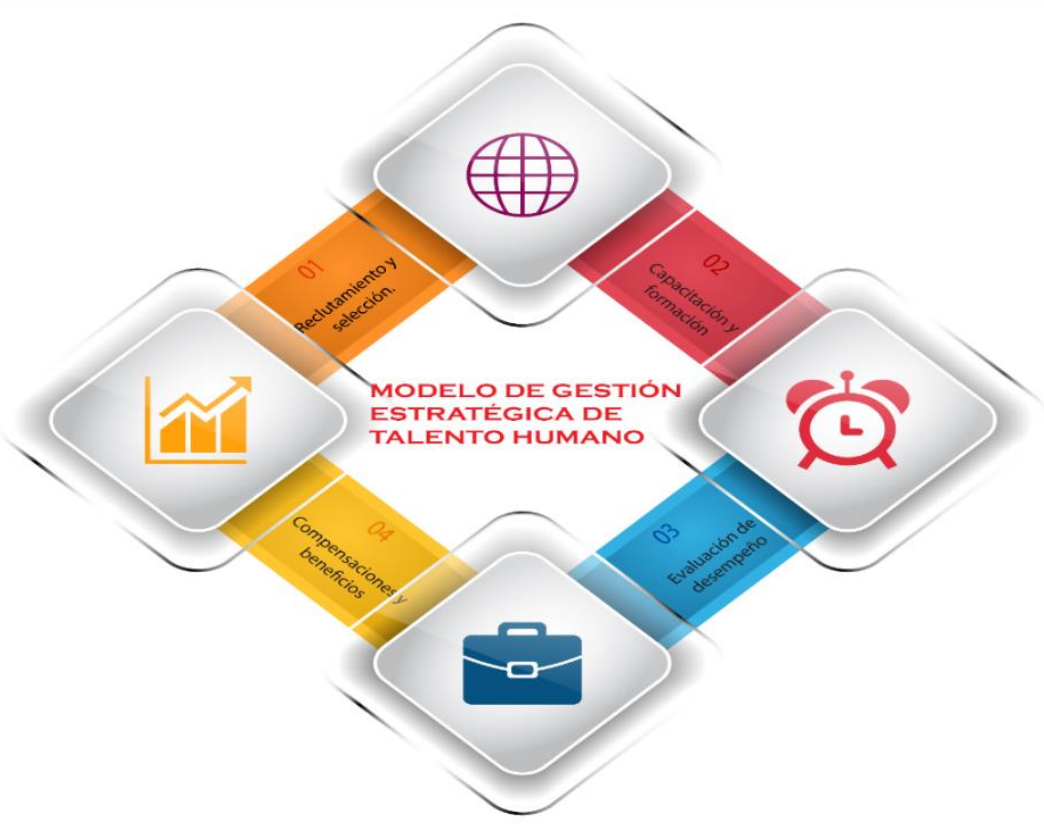

Figura 1. Propuesta de modelo de gestión estratégica de Talento Humano en el Instituto Ecuatoriano de Seguridad Social. 


\section{CIENCIAMATRIA}

Revista Interdisciplinaria de Humanidades, Educación, Ciencia y Tecnología

Año VII. Vol. VII. N¹2. Enero - Junio. 2021

Hecho el depósito de ley: pp201602FA4721

ISSN-L: 2542-3029; ISSN: 2610-802X

Universidad Nacional Experimental Francisco de Miranda (UNEFM). Santa Ana de Coro. Venezuela

Janeth Gabriela Molina-Romo; Katina Vanessa Bermeo-Pazmiño

Diego Patricio Cisneros-Quintanilla

\section{Reclutamiento y Selección: Análisis PEST}

Se indica un estudio del medio interno y externo de la Institución, mediante un análisis PEST, con el objetivo de conocer como diversos factores podrían afectar de manera positiva o negativa en el futuro desempeño de la unidad administrativa de talento humano, en el proceso de reclutamiento y selección de personal (Corrales-Morales, 2009).

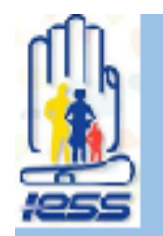

Asamblea legislativa: al registro y aprobación de cualquier ley que se relaciones con la salud, y en especial del talento humano.

Ministerio de Trabajo: reglamentos, acuerdos, resoluciones y plataformas virtuales que afecten directamente al proceso de selección y reclutamiento de personal.

Legislación laboralilos procesos se basan en gestionar lo

Fuerzas relacionado con los trabajadores y empleados, por lo que políticasy cualquier cambio en las leyes debe ser adoptado legales: inmediatamente por la Institución.

Ministerio de Finanzas: directrices que se emita en torno a los salarios y aumentos salariales por ley de igual manera políticas que se adopte por la austeridad del país.

Estrategias Organizacionales del IESS: la estrategia que formule el IESS en referencia al reclutamiento y selección de personal, y brechas de talento humano, son de estricto cumplimiento con la finalidad de que se logre los objetivos organizacionales.

\section{P E \\ S T}

La actitud de los empleados hacia el trabajo que puede af ectar el desempeño de la unidad administrativa de talento humano.

La nueva modalidad del teletrabajo hace que los colaboradores

Fuerzas no sean supervisados por sus jefes de manera directa, pues iociocultura laboran desde sus casas. Es importante al realizar un proceso de les selección, buscar candidatos con valores de responsabilidad, nrden nuntılalidar

Se debe considerar la comunicación que existe entre el jefe inmediato y el director para los requerimientos de personal acorde a las necesidades Institucionales
La situacion economica que atraviesa el pais y el munao

af ecta directamente al Instituto Ecuatoriano de

Seguridad Social por ende a los funcionarios y trabajadores.

Si la situación es positiva se podría planificar la ejecución de concursos de méritos y oposición para estabilizar al personal, o contratar personal que sea necesario en la Institución.

Si la situación es negativa la supresión de puestos, plazas que afectan directamente a la Institución.

Fuerzas

Económicas

Los cambios tecnológicos en los sistemas de talento humano, la Institución debe estar a la vanguardia en cuanto a los sistemas y plataformas que maneja talento humano. tecnológicas

Mantener actualización constante en los softwares de tal manera que todos los procesos en especial de reclutamiento y selección de personal.

Figura 2: Análisis PEST.

Fuente: Elaboración Propia. 


\section{CIENCIAMATRIA}

Revista Interdisciplinaria de Humanidades, Educación, Ciencia y Tecnología

Año VII. Vol. VII. N¹2. Enero - Junio. 2021

Hecho el depósito de ley: pp201602FA4721

ISSN-L: 2542-3029; ISSN: 2610-802X

Universidad Nacional Experimental Francisco de Miranda (UNEFM). Santa Ana de Coro. Venezuela

Janeth Gabriela Molina-Romo; Katina Vanessa Bermeo-Pazmiño

Diego Patricio Cisneros-Quintanilla

\section{Capacitación y formación}

Se puede evidenciar que en años anteriores no se realizó un levantamiento de información adecuada de todos los funcionarios que requerían formarse y capacitarse para mejorar sus habilidades, técnicas y recordar sus estrategias institucionales, por área.

\section{Tabla 1.}

Ejecución de capacitaciones de los años 2018-2019.

\begin{tabular}{|c|c|c|c|c|}
\hline $\begin{array}{l}\text { Á reas que se } \\
\text { capacitaron }\end{array}$ & $\begin{array}{l}\text { Tema de capacitación } \\
2018\end{array}$ & Tema de capacitación 2019 & $\begin{array}{c}\text { Número de } \\
\text { Funcionarios } 2018\end{array}$ & $\begin{array}{c}\text { Número de } \\
\text { funcionarios } \\
2019 \\
\end{array}$ \\
\hline Administrativo & $\begin{array}{l}\text { Administradanes de } \\
\text { Contrato }\end{array}$ & 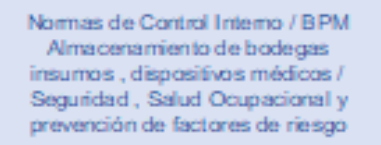 & 1 & 5 \\
\hline Fammacia & $\begin{array}{l}\text { Admiristradares de } \\
\text { Contrato }\end{array}$ & Mafivación y Relaciones Humaras & 1 & 1 \\
\hline Odantolog is & $\begin{array}{l}\text { Administradares de } \\
\text { Cortralo }\end{array}$ & Mafivación y Relsoiones Hurnaras & 2 & 2 \\
\hline Enfermeria & $\begin{array}{l}\text { Administradones de } \\
\text { Contrato }\end{array}$ & 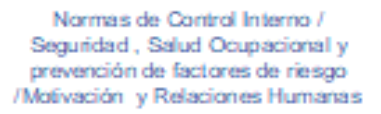 & 1 & 4 \\
\hline Rehisbiltacoión & $\begin{array}{l}\text { Administradares de } \\
\text { Contrato }\end{array}$ & $\begin{array}{l}\text { Seguridad, Sslud Doupsoional y } \\
\text { prevercoián de factores de riesgo }\end{array}$ & 1 & 1 \\
\hline Firnanciero & & Normas de Control lnterno & & 1 \\
\hline Trabajo Socisi & & Mativación y Relsoiones Hurnaras & & 1 \\
\hline Psicologia & & Mafivación y Relaciones Humaras & & 1 \\
\hline Admisianes & & Mativación y Relaciones Humarnas & & 1 \\
\hline Mexdicina Gerneral & & Mafivación y Redaciones Humaras & & 2 \\
\hline Terapia de Lenguaje & & $\begin{array}{l}\text { Segunidad, Sslud Ooupscionsal y } \\
\text { prevernoián de factores de riesgo }\end{array}$ & & 1 \\
\hline Terapis acupsciandi & & $\begin{array}{l}\text { Segunidad, Sslud Ocupscional y } \\
\text { prevernoián de factores de riesgo }\end{array}$ & & 1 \\
\hline \multirow[t]{3}{*}{ Talernto Humano } & & $\begin{array}{l}\text { Segguridad, Salud Ooupsoional y } \\
\text { prevencián de factores de riesgo }\end{array}$ & & 1 \\
\hline & & Total de funcionarios capsoitadas & 6 & 22 \\
\hline & & $\begin{array}{l}\text { Total de tunclonarlos de la Unilad } \\
\text { Medlica }\end{array}$ & & 65 \\
\hline
\end{tabular}

Fuente: Unidad Administrativa de Talento Humano del CRIE. 


\section{CIENCIAMATRIA}

Revista Interdisciplinaria de Humanidades, Educación, Ciencia y Tecnología

Año VII. Vol. VII. No12. Enero - Junio. 2021

Hecho el depósito de ley: pp201602FA4721

ISSN-L: 2542-3029; ISSN: 2610-802X

Universidad Nacional Experimental Francisco de Miranda (UNEFM). Santa Ana de Coro. Venezuela

Janeth Gabriela Molina-Romo; Katina Vanessa Bermeo-Pazmiño

Diego Patricio Cisneros-Quintanilla

Se propone el uso del siguiente formulario que será aplicado a todas las áreas de la institución para el levantamiento de capacitaciones específicas de todos los funcionarios.

\section{Tabla 2.}

Requerimiento de capacitaciones para la institución.

INSTITUTO ECUATORIANO DE SEGURIDAD SOCIAL

CENTRO DE REHABILITACIÓN INTEGRAL ESPECIALIZADO AZOGUES

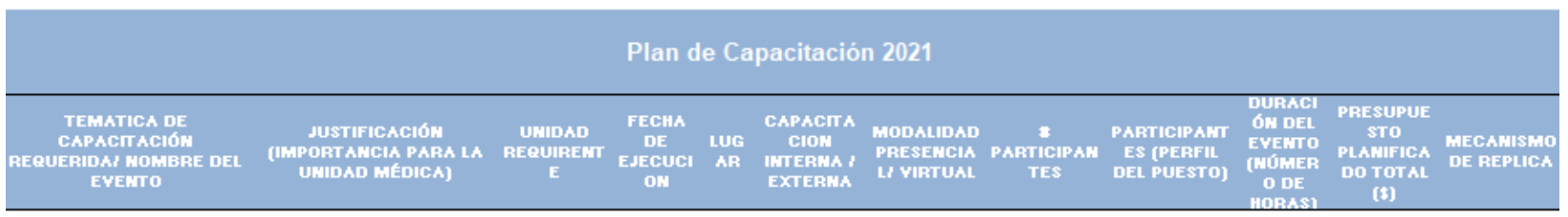

Elaborado por:

Autorizado por:

Fuente: Elaboración propia.

La Institución cuenta con un Plan Institucional y el Instructivo para el Procedimiento de Formación y Capacitación 2020, sin embargo, este plan institucional se realiza a nivel nacional, a través de esta matriz donde es remitida a las UATH de las Unidades Médicas para su envío. Se pretende que con esta información cada departamento de la Institución levante sus necesidades de capacitaciones, para luego ser expuestas con la unidad de talento humano y remitidas a planta central, de tal manera que puedan ser incluidas en el plan institucional nacional.

Puede servir de guía para gestionar los recursos económico y ejecución de las capacitaciones de la Institución, es importante mencionar que este levantamiento de información debe cumplir con tiempos y plazos establecidos por todas las áreas. 


\section{CIENCIAMATRIA}

Revista Interdisciplinaria de Humanidades, Educación, Ciencia y Tecnología

Año VII. Vol. VII. N¹2. Enero - Junio. 2021

Hecho el depósito de ley: pp201602FA4721 ISSN-L: 2542-3029; ISSN: 2610-802X

Universidad Nacional Experimental Francisco de Miranda (UNEFM). Santa Ana de Coro. Venezuela

Janeth Gabriela Molina-Romo; Katina Vanessa Bermeo-Pazmiño

Diego Patricio Cisneros-Quintanilla

El plan debe encontrase listo para el desarrollo y ejecución dentro de la Institución, cuyos conocimientos adquiridos en dichas capacitaciones deben ser replicados a cargos que tengan los mismos conocimientos técnicos y conductuales.

Evaluación de desempeño

El Ministerio de trabajo expidió mediante Noma Técnica del Subsistema de Evaluación del Desempeño, emitida mediante Acuerdo Ministerial No. MDT-2018-0041. Publicado en el Suplemento del Registro Oficial No. 218 de 10 de abril 2018 (Ministerio de Trabajo, 2018), para todos los funcionarios públicos amparados bajo el régimen de la LOSEP, el procedimiento de evaluación de desempeño consta 13 formularios.

En la nueva modalidad de evaluación los funcionarios carecen de preparación, conocimiento, y manejo escaso de herramientas tecnológicas, es así como se propone el siguiente flujograma que detallará paso por paso los procesos a seguir:

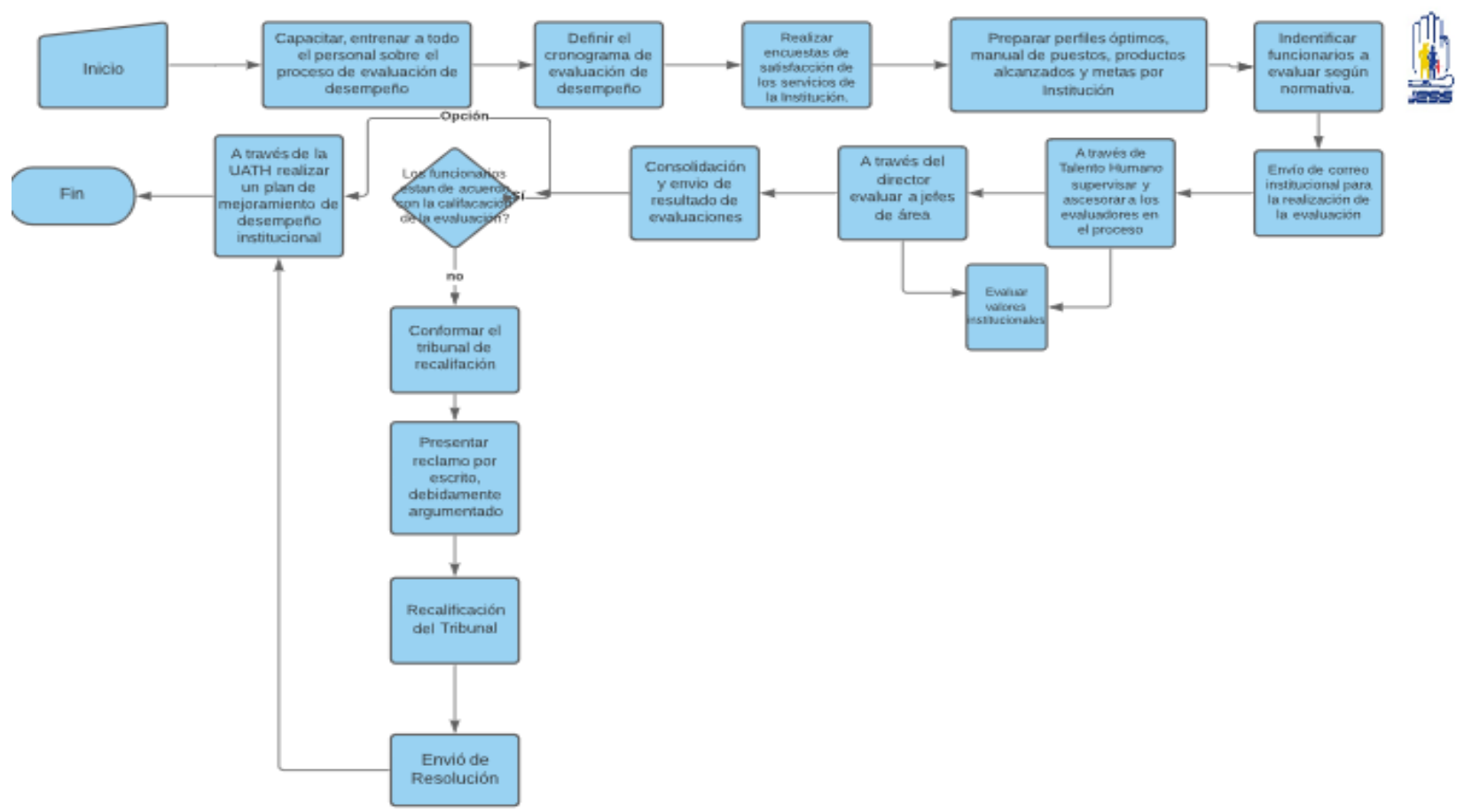

Figura 3. Proceso de Evaluación de desempeño propuesto.

Fuente: Elaboración Propia. 


\section{CIENCIAMATRIA}

Revista Interdisciplinaria de Humanidades, Educación, Ciencia y Tecnología

Año VII. Vol. VII. N¹2. Enero - Junio. 2021

Hecho el depósito de ley: pp201602FA4721

ISSN-L: 2542-3029; ISSN: 2610-802X

Universidad Nacional Experimental Francisco de Miranda (UNEFM). Santa Ana de Coro. Venezuela

Janeth Gabriela Molina-Romo; Katina Vanessa Bermeo-Pazmiño

Diego Patricio Cisneros-Quintanilla

\section{Compensaciones y beneficios.}

Este modelo de gestión pretende implementar un manual de incentivos, para contribuir con el bienestar social, psicológico y espiritual de los funcionarios y trabajadores de la institución. El afán es crear condiciones favorables para el desarrollo de trabajo en el desempeño laboral, cumplir con los objetivos institucionales y reconocer o premiar a los funcionarios y trabajadores que han contribuido con la planificación estratégica en el cumplimiento de objetivos del IESS. A continuación, se presenta una tabla en la que consta la estructura del plan de incentivos para el personal de la institución: 


\section{CIENCIAMATRIA}

Revista Interdisciplinaria de Humanidades, Educación, Ciencia y Tecnología

Año VII. Vol. VII. Nº12. Enero - Junio. 2021

Hecho el depósito de ley: pp201602FA4721

ISSN-L: 2542-3029; ISSN: 2610-802X

Universidad Nacional Experimental Francisco de Miranda (UNEFM). Santa Ana de Coro. Venezuela

Janeth Gabriela Molina-Romo; Katina Vanessa Bermeo-Pazmiño

Diego Patricio Cisneros-Quintanilla

\section{Tabla 3.}

Plan de Incentivos para funcionarios y trabajadores del IESS.

\section{Propuesta de Plan de Incentivos}

\begin{tabular}{|c|c|c|}
\hline $\mathbf{N}^{\circ}$ & Actividades & Descripción \\
\hline 1 & Resumen Ejecutivo & $\begin{array}{l}\text { Compuesto por los principios de: humanización del } \\
\text { trabajo, respeto, inclusión vocación de servicio, } \\
\text { compromiso, integridad, justicia, lealtad, servicio. }\end{array}$ \\
\hline 2 & Objetivo General & $\begin{array}{l}\text { Motivar, estimular, reconocer y premiar el desempeño } \\
\text {, de aquellas calificación de sobresaliente de forma } \\
\text { individual o en equipos de trabajo de la Institución }\end{array}$ \\
\hline 3 & Objetivos Especificos & $\begin{array}{c}\text { Conformado por } 5 \text { objetivos específicos relacionados } \\
\text { con el objetivo general. }\end{array}$ \\
\hline 4 & Alcance & Se beneficiarán todos los funcionarios de la institución \\
\hline 5 & $\begin{array}{l}\text { Conformación del comité de } \\
\text { Incentivos }\end{array}$ & $\begin{array}{l}\text { Seran integrantes: } \\
\text { Director Técnico de la institución o su delegado } \\
\text { Responsable de la U nidad Adminsitrativa de Talento } \\
\text { Humano o su delegado. }\end{array}$ \\
\hline & & $\begin{array}{l}\text { Representante democrático de servidores públicos } \\
\text { Representante democrático de trabajadores }\end{array}$ \\
\hline 6 & $\begin{array}{l}\text { Obligaciones del comité de } \\
\text { Incentivos }\end{array}$ & $\begin{array}{l}\text { Estará conformado por } 10 \text { items en beneficio de la } \\
\text { funcionarios y trabajadores. }\end{array}$ \\
\hline 7 & $\begin{array}{l}\text { De las funciones de los miembros } \\
\text { del comité }\end{array}$ & $\begin{array}{l}\text { En este paso se describe todas las funciones de : } \\
\qquad \begin{array}{l}\text { Presidente } \\
\text { Miembros } \\
\text { Secretario }\end{array}\end{array}$ \\
\hline 8 & Actividades & $\begin{array}{c}\text { Señala: } \\
\text { Términos para desarrollar el proceso de evaluación y } \\
\text { entrega de incentivo individual. } \\
\text { Requisitos para otorgar un incentivo }\end{array}$ \\
\hline
\end{tabular}


CIENCIAMATRIA

Revista Interdisciplinaria de Humanidades, Educación, Ciencia y Tecnología

Año VII. Vol. VII. N¹2. Enero - Junio. 2021

Hecho el depósito de ley: pp201602FA4721

ISSN-L: 2542-3029; ISSN: 2610-802X

Universidad Nacional Experimental Francisco de Miranda (UNEFM). Santa Ana de Coro. Venezuela

Janeth Gabriela Molina-Romo; Katina Vanessa Bermeo-Pazmiño

Diego Patricio Cisneros-Quintanilla

\begin{tabular}{|c|c|c|}
\hline \multirow[t]{2}{*}{9} & Estímulos & $\begin{array}{c}\text { Económicos } \\
\text { Dependen directamente de las autoridades centrales } \\
\text { del IESS } \\
\text { Reconocimiento } \\
\text { Carta de felicitación a ser induida en el expediente } \\
\text { laboral } \\
\text { Capacitación y formación }\end{array}$ \\
\hline & & Participación adiva con los objetivos de la institución \\
\hline 10 & Responsable de Aplicación & $\begin{array}{c}\text { Responsable de la Unidad Adminsitrativa de Talento } \\
\text { Humano o su delegado. } \\
\text { Responsables de cada área } \\
\text { Miembros del comité de incentivos }\end{array}$ \\
\hline 11 & Condusiones y recomendaciones & \\
\hline
\end{tabular}

Fuente: Elaboración Propia.

Esta propuesta pretende además mejorar el clima laboral actual, ya que al analizar las encuestas el personal de la institución requiere reconocimiento a su trabajo, que influiría directamente en sus objetivos personales e institucionales, capaces de llenar sus expectativas.

\section{CONCLUSIONES}

Se determinó un Modelo de gestión estratégica de Talento Humano para el Instituto Ecuatoriano de Seguridad Social, con la finalidad de mejorar el desarrollo de todos los subsistemas que se encuentran alienados a la planificación estratégica institucional basado en cuatro ejes: Reclutamiento y selección; capacitación y formación, Evaluación de desempeño y compensaciones y beneficios.

En el proceso de aplicación de las encuestas se constató que el personal reconoce a la Unidad Administrativa de Talento Humano como un proceso estratégico, sin embargo, no cubre por completo sus necesidades. Se evidenció que la Institución, posee una estructura organizacional definida, bajo lo cual desarrolla los procesos.

Sin embargo, el retraso de procesos se debe en varios casos al déficit de capacitaciones específicas acorde funciones que desempeñan, al crear estrategias que mejoren este 
proceso se obtendrían servidores públicos altamente capacitados mejorando su productividad para alcanzar la planificación estratégica.

Además, al incorporar este modelo mejoraría el clima laboral actual ya que se incentivaría y reconocería el esfuerzo adicional que realizan los funcionarios en la ejecución de actividades repercutiendo directamente en la calidad y eficacia de la atención hacia los afiliados, jubilados y pensionistas.

\section{REFERENCIAS CONSULTADAS}

Aduna-Mondragón, A, García- Mata, E, \& Chávez-Gutiérrez, E. (2017). Modelos de gestión de Recursos Humanos [Human Resources management models]. TEPEXI Boletín Científico De La Escuela Superior Tepeji Del Río, 4(8). https://doi.org/10.29057/estr.v4i8.2384

Alles, M. (2008). Naturaleza de los Individuos. En M. Alles, Comportamiento Organizacional. Como lograr un cambio cultural a través de la gestión por competencias (p. 33). Buenos Aires: Ediciones Granica S.A.

Armijos-Mayon, F, Bermúdez-Burgos, A, \& Mora-Sánchez, N. (2019). Gestión de administración de los Recursos Humanos [Management of Human Resources]. Revista Universidad y Sociedad, 11(4), 163-170.

Barrios-Hernández, K, Olivero-Vega, E, \& Figueroa-Saumet, B. (2020). Condiciones de la gestión del talento humano que favorecen el desarrollo de capacidades dinámicas [Conditions of human talent management that favor the development of dynamic capabilities]. Información tecnológica, 31(2), 55-62. https://dx.doi.org/10.4067/S0718-07642020000200055

Bustos-Farías, E, Cerecedo-Mercado, M, \& García-González, M. (2016). Modelo de gestión de conocimiento para el desarrollo de posgrado: estudio de caso [A Knowledge Management Model for Graduate Development: a case study]. Revista electrónica de investigación educativa, 18(1), 128-139.

Cerón-Gordón, J, Atiencia-Aucancela, E, López-Aguirre, J, Fernández-Vinueza, D, \& Llangara-Arellano, V. (2017). Estrategia de fortalecimiento en el talento humano [Strategy for strengthening human talent]. CE Contribuciones a la Economía. Recuperado de https://n9.cl/ehz5b 
Chávez, L. V. (2015). Gestión del Talento Humano [Human talent management]. Riobamba: La Caracola Editores.

Chiavenato, I. (2002). Gestión del Talento Humano [human talent management]. México: McGraw Hill.

Chiavenato, I. (2004). Introducción a la Teoría General de la Administración (Séptima Edición ed.). México: McGraw-Hill Interamericana.

Chiavenato, I. (2009). Gestión de Talento Humano. México: Mc Graw-Hill.

Chiavenato, I., \& Sapiro, A. (2017). Planeación estratégica. Fundamentos y Aplicaciones [Strategic planning. Fundamentals and Applications] (Tercera edición ed.). México D.F: MCGRAW-HILL INTERAMERICANA EDITORES, S.A. de C.V.

Corrales-Morales, D. (2009). Estrategia de Recursos Humanos de Avantek del Norte S.A. http://hdl.handle.net/2238/2832

Delaux, H. S. (2017). Modelo de Gestión Estratégica de RRHH en Organizaciones del Estado [Model of Strategic HR Management in State Organizations]. Europa: Redactum.

Ley de Seguridad Social. (2011). Registro Oficial Suplemento 465 de 30-nov-2001. Recuperado de https://n9.cl/zdvc

Ley Orgánica del Servicio Público, LOSEP. Registro Oficial Suplemento 294 de 06-oct.2010. https://n9.cl/85zgn

Marín-Samanez, H, \& Placencia-Medina, M. (2017). Motivación y satisfacción laboral del personal de una organización de salud del sector privado [Work motivation and job satisfaction of a private health care organization staff]. Horizonte Médico (Lima), 17(4),

52. https://dx.doi.org/https://doi.org/10.24265/horizmed.2017.v17n4.08

Pérez-Montejo, A. (2009). Evaluación del desempeño laboral [Job performance evaluation]. UPIICSA XVII, VII, 50-51. 


\section{CIENCIAMATRIA}

Revista Interdisciplinaria de Humanidades, Educación, Ciencia y Tecnología

Año VII. Vol. VII. N¹2. Enero - Junio. 2021

Hecho el depósito de ley: pp201602FA4721

ISSN-L: 2542-3029; ISSN: 2610-802X

Universidad Nacional Experimental Francisco de Miranda (UNEFM). Santa Ana de Coro. Venezuela

Janeth Gabriela Molina-Romo; Katina Vanessa Bermeo-Pazmiño

Diego Patricio Cisneros-Quintanilla

Pico, L. M. (2016). La gestión del talento humano, recurso indispensable para la organización en el entorno competitivo actual [The management of human talent, an indispensable resource for the organization in today's competitive environment]. INNOVA Research Journal, 1(11), 97-104. https://doi.org/10.33890/innova.v1.n11.2016.122

Quesada-Quecan, L. (2014). Gestión del talento humano, como promotor del desarrollo organizacional. [Management of the human talent, as promoter of the development organizacional]. Recuperado de http://hdl.handle.net/10654/11842

Stoner, J. \& Wankel, Ch. (1990). Administración. Prentice may. México.

Ulrich, D. (2000). Recursos Humanos Champions: cómo pueden los Recursos Humanos cobrar valor y producir resultados. Buenos Aires: Granica.

Werther, W. B. (2016). Administración de Personal [Personnel Administration]. México: McGraw Hill.

Zuin-Secco, F, \& França-da-Cunha, C, \& Spers, E, \& Galeano, R, \& Ragazzo-Corrêa-daSilva, R. (2016). Etapas de la planificación estratégica de marketing y el ciclo de vida organizacional: estudio de una empresa de gestión familiar [Stages of strategic marketing planning and the organizational life cycle: study of a family-run company]. Invenio, 19(36),65-87.

(C2021 por los autores. Este artículo es de acceso abierto y distribuido según los términos y condiciones de la licencia Creative Commons Atribución-NoComercial-Compartirlgual 4.0 Internacional (CC BY-NC-SA 4.0) (https://creativecommons.org/licenses/by-nc-sa/4.0/). 